\title{
Sorting system study based on the stochastic and Exhaustive Parallel Limited-1 polling control theory
}

\author{
Wenxue RAN ${ }^{1, a}$, Chiming $\mathrm{ZHAO}^{2, \mathrm{~b}}$, Fan $\mathrm{CHEN}^{3, \mathrm{c}}$ \\ ${ }^{1}$ Department of Logistics, Yunnan University of Finance and Economics, Kunming, 650221, China \\ ${ }^{2}$ Department of Logistics, Yunnan University of Finance and Economics, Kunming, 650221, China \\ ${ }^{3}$ Department of Logistics, Yunnan University of Finance and Economics, Kunming, 650221, China \\ aemail:ranwxa@vip.sina.com, bemail:chiming_zhao@163.com, \\ cemail:chenfan_19910725@126.com
}

Keywords: Exhaustive Parallel Limited-1; Polling Theory; Comparative Analysis

\begin{abstract}
The composite cigarette sorting system efficiency depends on a combined configuration of high flow sorting units and general sorting ones. This article innovatively proposes the polling theory of unit materials based on the actual demand for order picking under the herein scale. Put forward Exhaustive Parallel Limited-1polling theory as the basic control mechanism, this article analysis the control strategy of VC queue for priority orders and common ones, establishes the cost model of automatic Exhaustive Parallel Limited-1polling line, and also solving the related performance parameters. By the cost analysis between stochastic and Exhaustive Parallel Limited-1polling line system, it is easy to reach the conclusion that the polling theory is much benefit for cost savings. And a numerical example has been given to verify the effectiveness of the proposed method.
\end{abstract}

\section{Introduction}

At present, most Chinese distribution centers, such as cigarette distribution center, are use the stochastic sorting control technology. Fixed channel stochastic sorting control can overcome resource consumption problems. However, when business volume increases, especially under the e-commerce mode, the development of the logistics operation mode can not meet the rapidly expanding needs of customer, it is likely to result in the congestion of orders in process, so that the mean waiting time for orders increases, resulting the decrease of sorting system capabilities. Therefore, logistics and distribution centers need a new control mechanism to meet the expanding needs for large flow sorting business.

Scholars have done a lot of research on how to optimize the sorting problem. Gademan etc. [1, 2] studied the nearby sorting batches problems at the time of the batch service, and minimized the maximum lead time in any order, presented to use branch and bound method to solve the problem of moderate quantity orders sorting. While for large flow orders problem, he proposed the iterative approximation method. Mowrey and Parikh [3] presented a design of hybrid wide channel configuration, also developed the analysis model considering the stochastic storage, routing policy space and travel time on the basis of the traditional orthogonal channel. And through the cost optimization model to determine the system parameters, it's easily to obtain conclusions that mixed wide channel configuration is optimal. Under the specified storage strategy in the distribution center sorting area, Xiongzhi Wang et al [4] considered for replenishment operation problem, proposed the heuristic algorithm for complexity is $\mathrm{O}$ (n3), and simulated the performance of test algorithms, which works very well; Wei Xiao and Yaohua $\mathrm{Wu}$, etc. $[5,6]$ established a sorters portfolio optimization model under the minimization of unit sorting cost, so it can meet the optimal configuration of sorters.

However, the current domestic and foreign scholars mostly confined to the application of stochastic programming theory. In order to improve sorting efficiency, scholars began to use polling sorting system theory to study the sorting system. Kavitha and Combes [7] used the known 
pseudo-steady-state conservation system performance parameters in discrete voting system, to study the optimal system design of mixed polling system. With the application in wireless link and optimization of distributed garbage sorting system, it verified the optimized path mixed polling system configuration; Chernova et al [8] proposed a single server polling system with two queues, its stability region depending on the distribution of service time adaptive service strategy and a certain moment under the primitive index. Gong and Koster [9] used the stochastic polling theory, to research the problems of e-commerce dynamic Picking System modeling and solve the dynamic Picking manual picking system latency. Dongfeng Zhao et al. [10, 11] took advantages of probability generating function, constructed and analyzed the multi-level threshold polling system under the MAC discrete-time control protocol model, and explored the polling system of secondary site threshold services priority queues.

From the above analysis of literatures, we can see the current studies still exist the following problems:

(1) The existing researches were mostly based on the artificial and semi-stochastic sorting mode to evaluate and optimize the existing control mechanism, but always overlooked the expansion research of control mechanism;

(2) Although the existing researches for polling in order sorting had certain theoretical exploration, but the application of the concept is limited to manual sorting, which is not comply with modern concepts, such as [7] and [8]. Therefore, polling should be further applied to the study of deepening automated sorting area.

Therefore, this paper innovatively combines polling theory and automated sorting line, proposes a new type of parallel two level exhaustive limited-1 polling control mechanism. In addition, this paper has a depth modeling analysis between stochastic and limited-1 polling system, and compares two sorting costs which has an important reference for the application of new control mechanism by specific examples.

\section{Polling automatic sorting model of unit materials}

\section{Exhaustive parallel limited-1 two level polling control model of unit material}

The control mechanism of Exhaustive Parallel Limited-1 two level polling control system (EPL) is as follows: If an order sorting polling system consists of one priority and $\mathrm{N}$ common VC queues, then the priority order VC queue and common ones should operate parallel, and the priority VC queue should use exhaustive service control strategy, while the common queues should adapt to limited-1 service control theory.

Besides, when sorting machine starts operating, if the common order VC queue requires sorting, then common orders sorting would operate parallel exhaustive limited-1 sorting service, after one order queue is finished, system will instantly turn to the priority order VC queues, and priority to service the VC queues for priority orders. Similar to the combination of stochastic sorting line, the equipment group polling sorting line includes large flow sorting units and common ones, both of them consider the relationship between efficiency and varieties.

Units material exhaustive parallel limited-1 two level automated polling sorting line model and parameters

This paper focuses on fast, real-time and fairness sorting requirements of unit material, by in-depth research for the model, and applies theory to the sorting control strategies. Set steady state conditions of the established polling system model as $\sum_{\mathrm{i}=1}^{n_{2}} \lambda_{\mathrm{i}} \beta_{i}+\lambda_{h} \beta_{h}<1, i=1,2, \ldots, n_{1}$, the same as $\mathrm{n}_{2} \lambda \beta+\lambda_{h} \beta_{h}<1$. Under this case, when time of large flow sorters is set to be $t_{n^{*}}$, the probability generating function of the system state variables can be easily obtained as formula 1:

$$
G_{i h}\left(\mathrm{z}_{1}, z_{2}, \ldots, z_{i}, \ldots, z_{n_{2}}, z_{h}\right)=\lim _{n \rightarrow \infty} E\left(\prod_{\substack{j=1 \\ j \neq 1}}^{n_{2}} z_{j}^{\xi_{j}\left(n^{*}\right)} \cdot z_{h}^{\xi_{h}\left(n^{*}\right)}\right)
$$


$=R_{\mathrm{i}}\left(A_{h}\left(z_{h}\right) \prod_{j=1}^{n_{1}}\left(A_{j}\left(z_{j}\right)\right)\left\{\frac{1}{z_{i}} B_{i}\left(A_{h}\left(z_{h}\right) \prod_{j=1}^{n_{1}}\left(z_{j}\right)\right)\left\lfloor\begin{array}{l}G_{i}\left(z_{1}, z_{2}, \ldots, z_{i}, \ldots, z_{n_{2}}, z_{h}\right) \\ -G_{\mathrm{i}}(1,1, \ldots, 0, \ldots, 1,1)\end{array}\right]+G_{i}(1,1, \ldots, 0, \ldots, 1,1)\right\}\right.$

Among them, $i=1,2, \cdots, n_{1} ; j=1,2, \cdots, \mathrm{n}_{1}$.

When time of common sorters is $t_{n+1}$, and it is sorting order $i+1$, the probability generating function of the system state variables will be as formula 2 :

$$
\begin{aligned}
G_{i+1}\left(z_{1}, z_{2}, \cdots, z_{i}, \cdots, z_{\mathrm{n}_{1}}, z_{h}\right) & =\lim _{n \rightarrow \infty} E\left(\prod_{\substack{j=1 \\
j \neq i}}^{\mathrm{n}_{1}} z_{j}^{\xi_{j}(n+1)} \cdot z_{h}^{\xi_{h}(n+1)}\right) \\
& =G_{\mathrm{ih}}\left(z_{1}, z_{2}, \ldots, z_{n_{1}}, B_{h}\left(\prod_{j=1}^{n_{1}} A_{j}\left(z_{j}\right) Q_{h}\left(\prod_{j=1}^{\mathrm{n}_{1}} A_{j}\left(z_{j}\right)\right)\right)\right.
\end{aligned}
$$

Among them, $i=1,2, \ldots, \quad n_{2} ; \mathrm{j}=1,2, \ldots, \quad n_{2}, Q_{\mathrm{h}}\left(z_{h}\right)=A_{h}\left(B_{h}\left(z_{h} Q_{h}\left(z_{h}\right)\right)\right)$.

Besides, the mean cyclic period (MCP) of the exhaustive parallel limited-1two level priority control system is as formula 3:

$$
T_{c}=\frac{\eta p}{1-\lambda \beta_{h}-\eta \lambda(\beta-r)}
$$

According to the above analysis, the mean queue length of priority orders (MQLOPO) for large flow sorters can be reached, and it can be expressed as formula 4:

$$
g_{h}(h)=\frac{\eta r_{h} \lambda_{h}\left(1-\lambda_{l} \beta_{h}\right)}{1-\lambda_{1} \beta_{h}-\eta_{1}(\lambda \beta-\lambda r)}
$$

And also the mean queue length of common orders (MQLOCO) is as formula 5:

$$
\begin{aligned}
& g_{i}(\mathrm{i})=\frac{1-\lambda_{h} \beta_{h}}{21-\lambda_{h} \beta_{h}-n_{2} \lambda \beta} \\
& \quad \times\left\{n_{2} r\left\lfloor 1+\frac{\lambda_{h}^{2} \beta_{h}^{2}}{\left(1-\lambda_{h} \beta_{h}\right)^{2}}+\frac{\lambda}{1-\lambda_{h} \beta_{h}-n_{2}(\beta-r)}\left(n_{2}(\beta-r)+\frac{\lambda \beta_{h}^{2}\left(\lambda_{h} \beta_{h}+n_{2} \lambda(\beta-r)\right.}{\left(1-\lambda_{h} \beta_{h}\right)^{2}}\right)\right\rfloor A^{\prime \prime}(1)\right. \\
& \quad+\frac{n_{2} \lambda^{2} r}{\left(1-\lambda_{h} \beta_{h}-n_{2} \lambda(\beta-r)\right)\left(1-\lambda_{h} \beta_{h}\right)^{2}} B^{\prime \prime}(1)+\frac{n_{2} r \lambda^{2} \lambda_{h} \beta_{h}^{2}}{\left(1-\lambda_{h} \beta_{h}-n_{2} \lambda(\beta-r)\right)\left(1-\lambda_{h} \beta_{h}\right)^{2}} B_{h}^{\prime \prime}(1) \\
& \quad+\frac{n_{2} \lambda^{2}}{1-\lambda_{h} \beta_{h}}\left\lfloor 1+\lambda_{h} \beta_{h}-\lambda_{h}^{2} \beta_{h}^{2}-\frac{n_{2} \lambda r\left(3-2 \lambda_{h} \beta_{h}\right)}{\left(1-\lambda_{h} \beta_{h}-n_{2} \lambda(\beta-r)\right)\left(1-\lambda_{h} \beta_{h}\right)} \mid R^{\prime \prime}(1)+\frac{n_{2} r \lambda^{2} \lambda_{h} \beta_{h}}{1-\lambda_{h} \beta_{h}}\right. \\
& \left.\quad+\frac{n_{2} \lambda\left(2 r\left(1-\lambda \beta-\lambda_{h} \beta_{h}\right)+2 n_{2} r \lambda^{2} \lambda_{h} \beta_{h}\left(\beta \quad r+r \lambda \lambda_{h} \beta_{h}\left(1 \quad A_{h} \beta_{h}\right)+\left(n_{2}-1\right)(r \lambda-2 r \lambda \beta)\right)\right.}{\left(1 \quad \lambda_{h} \beta_{h}+n_{2} \lambda(\beta-r)\right)\left(1-\lambda_{h} \beta_{h}\right)}\right\}
\end{aligned}
$$

Mean waiting time of common orders (MWTOPO) for large flow sorters is expressed as formula 6 :

$$
E\left(w_{h}\right)=\frac{g_{i h}(h, h)}{2 \lambda_{h} g_{\text {ih }}(h)}+\frac{\lambda_{h} B_{h}{ }^{\prime \prime}(1)}{2\left(1-\lambda_{h} \beta_{h}\right)}-\frac{A_{h}{ }^{\prime \prime}(1)}{2 \lambda_{h}^{2}\left(1+\lambda_{h} \beta_{h}\right)}
$$

Among them, two level original pitch of large flow sorters orders queue is as formula 7:

$$
\begin{aligned}
g_{\text {ih }}(\mathrm{h}, \mathrm{h})= & \frac{\mathrm{n}_{1} r \lambda}{1-\lambda_{h} \beta_{h}-\left(n_{1} \lambda \beta-n_{1} r \lambda\right)}\left[A_{h}^{\prime \prime} 1(\beta-\mathrm{r})+\lambda_{h}^{2}\left(B^{\prime \prime}(1)-R^{\prime \prime}(1)\right)\right] \\
& +\lambda_{\mathrm{h}}^{2} R \oplus A_{h}^{\prime \prime} \text { (1) }
\end{aligned}
$$

MWTOCO ordinary sorting unit orders queue (Mean Waiting Time of Priority Order, the order of priority queues average wait time) is:

Mean waiting time of priority orders(MWTOCO) can be expressed as formula 8:

$$
E\left(w_{i}\right)=\frac{g_{i}(i)}{\lambda T_{c}}-\frac{A^{\prime \prime}(1)}{2 \lambda^{2}}-\frac{1}{\lambda}
$$




$$
=\frac{1-\lambda_{h} \beta_{h}-n_{2} \lambda(\beta-r)}{n_{2} \lambda^{2} r} \times g_{i}(i)-\frac{A^{\prime \prime}(1)}{2 \lambda^{2}}-\frac{1}{\lambda}
$$

Cost analysis of unit material exhaustive parallel limited-1 two level automated polling sorting line

Using the aforementioned cost analysis method of stochastic combinations sorting line, per unit sorting cost of the established exhaustive parallel limited-1 two level automated polling sorting line is as follows:

$$
C_{p o l}=n_{1} C_{d}^{*}+n_{2} C_{p}^{*}+C_{b}+n_{1} C_{d e}^{*}+n_{2} C_{p e}^{*}
$$

Among them, $C_{d}^{*}$ is the fixed cost of one single large flow sorter; $C_{d e}^{*}$ is the variable cost of one single large flow sorter; $C_{p}^{*}$ is the fixed cost of one single common sorter; $C_{p e}^{*}$ is the variable cost of one single common sorter.

Thus, the optimization objective cost function $F_{p o l}$ of the unit material exhaustive parallel limited-1 two level automated polling sorting line can be expressed as formula 10:

$$
\begin{aligned}
\min F_{p o l}= & {\left[n_{1} C_{d}^{*}+n_{2} C_{p}^{*}+C_{b}+n_{1} C_{d e}^{*}+n_{2} C_{p e}^{*}\right] \frac{T_{c}}{Q}+n_{1} C_{d e}^{*} E\left(w_{h}\right) \cdot \frac{\varphi}{Q} } \\
& +{ }_{2} n C_{p e} E\left(w_{i}\right) \cdot \frac{(Q-\varphi)}{Q}
\end{aligned}
$$

Among them, $\varphi$ is the total orders quantity serviced by large flow sorters; While $Q$ is goods total amount.

\section{Stochastic control sorting model of unit materials}

In order to facilitate control of system, this paper divided the line sorters into large flow sorters and common sorters. To facilitate the transfer of cargo delivery, it sets same brand unit material of one order could be adjacent in packing, therefore, each sorter can has serial work, throughput of order sorting is the time of order sorting, which equals to the sum of sorters working time. Modeling and Analysis are followed.

\section{Assumptions}

To solve the issues aforementioned, this paper composes some assumptions based on the practical application: (1) A variety of goods occupies only one sorter; (2) Same unit costs of different stations; (3) No waiting replenishment in sorting process; (4) Sorters sorting speed should be calculated as average speed; (5) Sorting line facilities ability adapt to the various components, no influence ability exists.

\section{Unit cost optimization of unit material automatic stochastic sorting line}

From the literature [6], unit cost optimization function $F_{a}$ of unit material automatic stochastic sorting line can be obtained as formula 11 :

$$
\begin{aligned}
\min F_{a}= & \frac{1}{Q}\left[n_{1} C_{d}^{*}+n_{2} C_{p}^{*}+\left(C_{d e}^{*}-C_{p e}^{*}\right) \sum_{i=1}^{n_{1}} \delta_{i}+n_{2} C_{p e}^{*}+C_{b}\right] \\
& \left.\times\left[\left(T_{d}-T_{p}\right) \sum_{i=1}^{n_{1}} \delta_{i} \varphi_{i}+T_{p} Q-\sum_{i=1}^{n_{1}} \delta_{i} \varphi_{i}\right)+T_{e}\right]
\end{aligned}
$$

Among them

$$
C_{d}\left(=c_{d}+c_{s}\right)(1+\alpha) /(y d)+f_{d}
$$

$$
C_{p}\left(=c_{p}+t_{p} c_{s}\right)(1+\alpha) /(\quad y d)+f_{p} c_{f}
$$

$$
\begin{aligned}
C_{d e}^{*} & =f_{d e} c_{d e} \\
C_{p e}^{*} & =f_{p e} c_{p e} \\
T_{d} & =1 /\left(\beta_{h d} \cdot h_{d}\right)
\end{aligned}
$$




$$
\begin{aligned}
& T_{p}=1 /\left(\beta_{p} \cdot h_{d}\right) \\
& T_{e}=(e-1) t_{0} /\left(3600 \cdot h_{d}\right)
\end{aligned}
$$

Among them, $C_{d}^{*}$ is fixed cost of single large flow sorter; $C_{d e}^{*}$ is variable cost of large flow sorters; $C_{p}^{*}$ is fixed cost of common sorters; $C_{p e}^{*}$ is variable cost of common sorters; $\beta_{h d}$ is sorting speed of large flow sorters, unit/ per hour; $n_{1}$ is quantity of large flow sorters; $n_{2}$ is quantity of common sorters; $\beta_{p}$ is sorting speed of common sorters, unit/ per hour; $\varphi_{i}$ is orders quantity of priority goods $i ; h_{d}$ is sorting time of one day, hours/ per day; $t_{0}$ is interval time of orders; $\delta_{i}$ is a decision variable, when goods $i$ use sorters to work, $\delta_{i}=1$, or $\delta_{i}=0 ; T_{d}$ is time of large flow sorters sort out unit goods; $T_{p}$ is time of common sorters sort out unit goods; $T_{e}$ is total interval time of orders; $C_{a}$ is unit time total cost of units material automatic stochastic sorting line, RMB; $C_{d}$ is unit time sorting cost of single large flow sorters, RMB; $d_{y}$ is work time of facilities, day/per year; $c_{d}$ is cost of single large flow sorter, RMB/unit; $f_{d}$ is the occupied place of single large flow sorter, square meters; $l_{d}$ is the length of large flow sorter transfers, meter/unit; $c_{d e}$ is loss cost of single large flow sorter, RMB/ unit; $c_{s}$ is unit cost of order transfer, $\mathrm{RMB} /$ meter; $c_{f}$ is occupied space, $\mathrm{RMB} /$ square meter; $\alpha$ is the maintenance factor of facilities cost; $f_{d e}$ is loss of single large flow sorter, including energy loss, materials loss, friction loss and so on, unit; $f_{p e}$ is loss of single common sorter, including energy loss, materials loss, friction loss and so on, unit; $y$ is equipment depreciation period, year; $C_{p}$ is unit time sorting cost of single common sorter, RMB; $f_{p}$ is occupied space of single common sorter, square meters; $c_{p}$ is facility cost of single common sorter, RMB/unit; $l_{p}$ is length of common sorters transfers, meter/unit; $c_{p e}$ is loss cost of single common sorter, RMB/unit; $C_{b}$ is unit time sorting cost of second segment facilities, including transferring, coding, packing, control system and computer system, RMB; $f_{b e}$ is loss of second segment facilities, including transferring, coding, packing, control system and computer system, unit; $c_{b}$ is the cost of second segment facilities, including transferring, coding, packing, control system and computer system, RMB/unit; $c_{b e}$ is loss cost of second segment facilities, including transferring, coding, packing, control system and computer system, RMB/unit; $l_{b}$ is the length of second segment transfers, meter/unit; $r_{g}$ is number of employees, person; $f_{b}$ is occupied space of second segment facilities, including transferring, coding, packing, control system and computer system, square meters; $c_{r}$ is mean wage of sorting line employees, RMB/person.

\section{Numerical example}

Take a distribution center as an example, it operates whole city's sales orders sorting, facilities and personnel related data has been shown in Table 1, and orders quantity of each variety of goods has been shown in Table 2, total orders quantity is set for 107 (Note: 1 order $=20$ units), so set $n_{1}=2, n_{2}=8$, set equal sorting opportunities to 2 large flow sorters, using exhaustive service theory, opportunities for 8 common sorters are also equal, using exhaustive limited-1service control mechanism. 
Table 1. Sorting facilities and personnel factors

\begin{tabular}{ccc}
\hline Symbol and Paramenters & Symbol and Paramenters & Symbol and Paramenters \\
\hline $\mathrm{C}_{\mathrm{d}}: 150000 \mathrm{RMB} /$ unit & $\mathrm{C}_{\mathrm{p}}: 20000 \mathrm{RMB} /$ unit & $\mathrm{C}_{\mathrm{s}}: 3000 \mathrm{RMB} /$ meter \\
$\mathrm{l}_{\mathrm{d}}: 1.5 \mathrm{~meter} /$ unit & $\mathrm{l}_{\mathrm{p}}: 0.5$ meter / unit & $\mathrm{C}_{\mathrm{f}}: 0.5 \mathrm{RMB} /$ square meter \\
/day \\
$\mathrm{f}_{\mathrm{d}}: 0.5$ square meter / unit & $\mathrm{f}_{\mathrm{p}}: 0.2$ square meter / unit & $\alpha: 20 \%$ \\
$\beta_{\mathrm{hd}}: 12.5$ unit/second & $\beta_{\mathrm{p}}: 2.5$ unit/second & $\mathrm{t}_{0}: 2$ second/unit \\
$\mathrm{y}: 15$ year & $\mathrm{d}_{\mathrm{y}}: 250$ days /year & $\mathrm{C}_{\mathrm{r}}: 100 \mathrm{RMB} /$ person/day \\
$\mathrm{C}_{\mathrm{de}}: 48 \mathrm{RMB} /$ unit / day & $\mathrm{r}_{\mathrm{g}}: 4$ person & $\mathrm{h}_{\mathrm{d}}: 8$ hours/day \\
$\mathrm{f}_{\mathrm{de}}: 2$ unit & $\mathrm{C}_{\mathrm{pe}}: 12 \mathrm{RMB} /$ unit / day & $\mathrm{e}: 10$ unit \\
$\mathrm{f}_{\mathrm{pe}}: 8$ unit & $\mathrm{C}_{\mathrm{be}}: 222 \mathrm{RMB} /$ day & $\mathrm{f}_{\mathrm{b}}: 40$ square meters \\
$\mathrm{f}_{\mathrm{be}}: 10$ unit & $\mathrm{C}_{\mathrm{b}}: 500000 \mathrm{RMB}$ & $\mathrm{l}_{\mathrm{b}}: 22$ meters \\
\hline
\end{tabular}

Table 2. Orders quantity of each species (common case)

\begin{tabular}{ccccc}
\hline \multirow{2}{*}{ Number } & $\begin{array}{c}\text { Orders } \\
\text { construction /unit }\end{array}$ & $E\left(w_{h}\right)$ & $E\left(w_{i}\right)$ & $T_{c}$ \\
\hline 1 & $16(13,3)$ & 0.4705 & 9.6314 & 2.8917 \\
2 & $21(17,4)$ & 0.4444 & 9.9660 & 2.2578 \\
3 & $3(1,2)$ & 0.4877 & 9.5964 & 4.0191 \\
4 & $9(3,6)$ & 0.3812 & 9.7695 & 1.5686 \\
5 & $13(4,9)$ & 0.2272 & 10.2731 & 1.0832 \\
6 & $1(0,1)$ & NAN & 9.5335 & 6.6784 \\
7 & $1(1,0)$ & 0.5011 & NAN & 20.0027 \\
8 & $10(9,1)$ & 0.4962 & 9.5825 & 6.7045 \\
9 & $15(8,7)$ & 0.3371 & 10.3874 & 1.3652 \\
10 & $18(10,8)$ & 0.2842 & 9.6445 & 1.2079 \\
\hline
\end{tabular}

According to the data from Table1 to 2, it can obtain that:

$C_{d}^{*}=49.69 \quad \mathrm{RMB} /$ day/unit; $\quad C_{p}^{*}=6.98 \quad \mathrm{RMB} /$ day/unit; $\quad C_{d e}^{*}=48 \quad \mathrm{RMB} /$ day; $\quad C_{p e}^{*}=12$ $\mathrm{RMB} /$ day; $c_{b}=2821.12 \mathrm{RMB} / \mathrm{day} ; T_{d}=0.08 \mathrm{~seconds} / \mathrm{unit} ; T_{p}=0.4 \mathrm{~seconds} / \mathrm{unit} ; T_{e}=21.2 \mathrm{~seconds} / \mathrm{unit}$ 。

Using the above parameters, it can be easily calculated that, when $n_{1}=2, n_{2}=8$, orders data under common cases shown in Table 2 is clear, the stochastic sorting cost is $\min C_{a}=0.0156$ $\mathrm{RMB} /$ unit; While the polling sorting cost is $\min C_{p o l}=0.0152 \mathrm{RMB} / \mathrm{unit}$.

For further study of two sorting line characters, this paper research for the following orders, one are priority orders quantity is larger than common ones, while the others are opposite. Data has been shown in Table 3 to 4.

Table 3. Cases of priority orders quantity is larger than common ones

\begin{tabular}{ccccc}
\hline $\begin{array}{c}\text { Numb } \\
\text { er }\end{array}$ & $\begin{array}{c}\text { Orders } \\
\text { construction /unit }\end{array}$ & $E\left(w_{h}\right)$ & $E\left(w_{i}\right)$ & $T_{c}$ \\
\hline 1 & $12(10,2)$ & 0.4865 & 9.5864 & 4.0280 \\
2 & $11(9,2)$ & 0.4862 & 9.6678 & 4.0310 \\
3 & $14(10,4)$ & 0.4452 & 9.7437 & 2.2549 \\
4 & $13(11,2)$ & 0.4854 & 9.5915 & 4.0362 \\
5 & $16(11,5)$ & 0.4131 & 9.7745 & 1.8509 \\
6 & $15(10,5)$ & 0.4153 & 9.6582 & 1.8476
\end{tabular}




\begin{tabular}{ccccc}
7 & $13(10,3)$ & 0.4682 & 9.5376 & 2.8900 \\
8 & $10(9,1)$ & 0.4962 & 9.5072 & 6.7106 \\
9 & $7(6,1)$ & 0.4971 & 9.5554 & 6.6953 \\
10 & $11(11,0)$ & 0.4997 & NAN & 20.3280 \\
\hline
\end{tabular}

Table 4. Cases of priority orders quantity is smaller than common ones

\begin{tabular}{ccccc}
\hline $\begin{array}{c}\text { Numb } \\
\text { er }\end{array}$ & $\begin{array}{c}\text { Orders } \\
\text { construction /unit }\end{array}$ & $E\left(w_{h}\right)$ & $E\left(w_{i}\right)$ & $T_{c}$ \\
\hline 1 & $13(3,10)$ & 0.1653 & 9.8416 & 0.9826 \\
2 & $11(2,9)$ & 0.2329 & 10.2213 & 1.0845 \\
3 & $13(3,10)$ & 0.1665 & 10.6131 & 0.9843 \\
4 & $13(2,11)$ & 0.0973 & 12.3143 & 0.9021 \\
5 & $15(4,11)$ & 0.2842 & 25.2755 & 0.9067 \\
6 & $15(5,10)$ & 0.6708 & 29.2006 & 0.9948 \\
7 & $12(2,10)$ & 0.1692 & 11.0972 & 0.9855 \\
8 & $11(2,9)$ & 0.2329 & 10.5976 & 1.0836 \\
9 & $8(2,6)$ & 0.3823 & 9.8948 & 1.5675 \\
10 & $11(0,11)$ & NAN & 12.0450 & 0.9007 \\
\hline \multicolumn{5}{r}{} \\
\hline
\end{tabular}

Similarly, from data of Table 3 , results can be obtained: $\min C_{a}=0.00014 \mathrm{RMB} / \mathrm{unit}$, $\min C_{p o l}=0.01135 \mathrm{RMB} / \mathrm{unit}$.

From data of Table 4, results can be obtained: $\min C_{a}=0.04590$ $\mathrm{RMB} / \mathrm{unit}, \min C_{p o l}=0.0384152 \mathrm{RMB} / \mathrm{unit}$.

So this paper applies to the actual situation of logistics distribution center, innovative use polling theory into the automated sorting mechanism, by three comparative different cases, comparatively analysis the cost of two control mechanism, stochastic and polling, so it can reach the optimal sorting control mode under set appropriateness.

By analyzing those data for further research, the basic conclusions are as follows:

(1) Parallel limited-1two level polling control mechanism has a significant role in orders sorting area, and the MQL, MWT and stochastic control, were significantly reduced. However, under some specific cases, costs of two control mechanism have fluctuation in exhaustive parallel limited-1 two level polling control system (EPL). When priority orders are larger than common ones, sorting cost under EPL is much larger. Because under construction, orders are mostly sorted by large flow sorters, and common sorters appear idling, that means the idle cost is much smaller than energy loss cost, so the stochastic control has a better performance. While priority orders are smaller than the common ones, large flow sorters have idle situation, polling's advantages appeared, so in these cases, polling system has a better performance.

(2) For support requirements of priority queues have priority service, EPL has shown the best characteristics. In the environment of queues conversion, the MWT of system is the lowest, which means the EPL system has the highest priority for one level and also two level polling. But this conclusion only applies to the case of priority and common orders have the same quantity.

(3) Under the same assumptions, if priority orders and common ones quantity are close, or priority ones is far less than the common orders, then the exhaustive limited-1 two level polling system has the lowest cost and best performance; While under the opposite case, stochastic mode has the lowest cost and further economic effective.

\section{Conclusions}

This paper innovatively combines polling service concept with automated sorting line, use polling as a significant tool of resource allocation and efficiency configuration studies, give a 
pioneering research to improve sorting efficiency of the new direction, and made innovative research results as following aspects:

(1) Innovatively use polling mechanism to study the production process of units material, and establish the polling sorting theory system, obtain the corresponding algorithm. The value is to break the existing fixed channel sorting control and stochastic sorting control theory, propose a new idea for unit material sorting technology development;

(2) Innovatively present EPL theory, use embedded Markov chain and multi-dimensional probability generating function, to get the formulas for MWT, MCP and MQL, and also do some further numerical analysis.

(3) Expanding the application of order sorting system integration theory, comparatively study the unit material automatic stochastic sorting line and EPL sorting line, make the scheduling polling control model into a research tool and method which can be achieved.

Although this paper has achieved some results, because of its wide field involves, so many factors, and high mechanical requirements for facilities, so in specific practice during the operation, it still needs further study. In this paper, the polling services theory meets the efficient sorting for e-commerce needs of scale economy, so this is a valuable research direction.

\section{References}

[1] Gademann N, Van de Velde S. Batehing to minimize total travel time in a parallel- aisl warehouse. IIE Transaetions. 2005, 37(1): 63-75.

[2] Gademann A J R M, van den Berg J P, vander Hoff H H. an order batching algorithm for wave picking in a parallel-aisle warehouse. iie transactions. 2001, 33(5): 385-398.

[3] Mowrey C H, Parikh P J. mixed-width aisle configurations for order picking in distribution centers. european journal of operational research. 2014(1): 87-97.

[4] Xiongzhi Wang, Guoqing Wang. Heuristics of distribution center replenishment operation problem. System Engineering-Theory \& Practice. 2008: 50-56.

[5] Yigong Zhang, Yaohua Wu. Automatic sorting system items distribution based on parallel sorting strategy. Computer Integrated Manufacturing Systems. 2010: 1720-1725.

[6] Jiwei Xiao, Yaohua Wu, Shanzuo Lou, et al. Optimization of combinatorial hybrid cigarette sorting system. System Engineering-Theory \& Practice. 2010: 251-256.

[7] Kavitha V, Combes R. Mixed polling with rerouting and applications. Performance Evaluation. 2013, 70(11): 1001-1027.

[8] Chernova, Natalia, Foss, et al. a polling system whose stability region depends on the whole distribution of service times. operations research letters. 2013(2): 188-190.

[9] Gong Y, Koste R D. A polling-based dynamic order picking system for online retailers. IIE Transactions. 2008, 40(3): 1070-1082.

[10] Dongfeng Zhao,Hongwei Ding,Yifan Zhao, et al. MAC discrete time control protocol model analysis of multi-level threshold service polling system. Journal of Electronic. 2010: 1495-1499.

[11] Minggui Wang, Dongfeng Zhao, Hongwei Ding, et al. Polling system analysis of center queue secondary threshold service. Computer Engineering and Applications. 2009: 94-96. 